\title{
Opportunistic Reliability for Cognitive Radio Sensor Actor Networks in Smart Grid
}

\author{
Ozgur Ergula ${ }^{\mathrm{a}}$, A. Ozan Bicen ${ }^{\mathrm{b}}$, Ozgur B. Akan ${ }^{\mathrm{a}}$ \\ ${ }^{a}$ Next-generation and Wireless Communications Laboratory \\ Department of Electrical and Electronics Engineering \\ Koc University, Istanbul, 34450 Turkey \\ ${ }^{b}$ Broadband Wireless Networking Laboratory \\ School of Electrical and Computer Engineering \\ Georgia Institute of Technology, Atlanta, GA 30332 USA
}

\begin{abstract}
Reliability is one of the most important requirements in Smart Grid communications. Reliable detection of an emergency event enables timely response. Within the automated nature of Smart Grid, such detection and response are carried out by sensor and actuator nodes. Therefore, it is important to study the capabilities of wireless sensor actor networks. In this paper, we first present an analysis of reliability in sensor actor networks, and lay out the factors that effect reliability. We then propose a scheme, where actor nodes cooperate to reach a global estimate under interruptions due to licensed user interference, i.e., consensus. We show that consensus improves reliability compared to local estimation of event features. We further show that convergence rate depends on connectivity of actors. Our analyses are generic and can be applied to inhomogeneous licensed user activity and interference on channels. A simulation study is presented to support our analyses and demonstrate the performance of proposed scheme in achieving consensus and mitigating disagreement among actor nodes.
\end{abstract}

Keywords: Smart Grid communications, cognitive radio sensor networks, distributed sensing, spectrum sharing.

\section{Introduction}

Wireless sensor networks [1] and sensor actor networks [2] are expected to watch over expensive equipment in Smart Grid, with sensors monitoring the environment for possible emergency events and communicating with actuators to take proper action when the event occurs. Failure in detection of an emergency event may result in break down of expensive equipment, and in some extreme cases, may even cost lives. Therefore, reliability is considered to be one of the most important issues in Smart Grid communications. To avoid such losses reliable and timely coordination of sensing results must be addressed.

One of the biggest problems in wireless communications is spectrum scarcity [3]. The demand for wireless spectrum is growing rapidly and new paradigms such as Internet-of-Things and Device-to Device communications, which are expected to have massive spectrum demand are emerging. In Smart Grid communications, the situation is exacerbated by electromagnetic interference of the devices and harsh environmental conditions.

Cognitive radio sensor networks (CRSN) are proposed to mitigate spectrum scarcity specifically in dense deployed sensor networks and enable distributed sensing over temporally unoccupied portions of the licensed spectrum [4]. Incorporation of cognitive radio $(\mathrm{CR})$ into distributed sensing requires

Email addresses: ozergul@ku.edu.tr (Ozgur Ergul), bozan@ece.gatech. edu (A. Ozan Bicen), akan@ku. edu.tr (Ozgur B. Akan)

Preprint submitted to Ad Hoc Journal sharing of spectrum opportunities among CRSN nodes while addressing event specific sensing requirements, while adhering to the inherited collaborative and energy-constrained nature of sensor networks. Furthermore, cognitive radio actor nodes can collect observations of CRSN nodes and cooperate to reach a global estimate, i.e., consensus [5]. Reaching consensus in a decision, i.e., estimation, interval requires efficient and adaptive sharing of spectrum opportunities among cognitive radio sensor and actor nodes, such that desired amount of samples can be collected from sensors with minimum spectrum access and actors can reach a consensus during an estimation interval.

Due to limited battery sizes, energy efficiency has been the main consideration for communication algorithm design for CRSN. Energy-efficient channel management is studied in [6]. Residual energy aware channel assignment is investigated in [7]. Energy-efficient spectrum sensing algorithm for CRSN is proposed in [8]. However, these works mainly lack in incorporating collaborative nature of sensor networks, and application specific reliability, i.e., estimation distortion and interval, requirements are disregarded. Solutions that take correlations between sensor readings have been proposed to take advantage of sensor collaboration $[9,10,11]$ in WSN. These solutions should be modified to address opportunistic operation in a CRSN, taking spectrum conditions into account. Spectrum sharing problem for cognitive radio networks (CRN) are investigated in $[12,13,14,15,16]$. Main drawback of these schemes is missing consideration of detection and monitoring requirements of sensor networks. In our design, we specifically target to exploit collaborative effort of sensor nodes on the same event 
and achieve reliability opportunistically while reaching consensus among actor nodes in an estimation interval.

Spectrum sharing solutions in other CRN mainly focus on throughput maximization and are proposed to efficiently utilize the scarce spectrum opportunities. In [12], spectrum sharing for multi-hop cognitive radio networks is studied. In $[13,14,15,16]$ spectrum sharing schemes for multiple input multiple output systems have been proposed. The primary reason for their inapplicability in CRSN is their notion of rate maximization. Furthermore, reliable estimation of event features, collaborative nature of CRSN nodes, coordination requirements of $\mathrm{CR}$ actor nodes and spectrum efficiency must be addressed while designing spectrum management algorithms for large scale distributed sensing networks. Hence, there is a need for a novel spectrum access mechanism in CRSN that emphasizes on collective reliability, spectrum efficiency and simplicity.

CR based communication in Smart Grid has been investigated in various fronts, such as quaity-of-service (QoS) or priority based access schemes [17, 18], network infrastructure proposals [19], investigation of important parameters in CR based network design [20], multimedia communications [21], and green communications [22]. However, reliability is not investigated thoroughly, even though it is one of the most important requirements.

In this paper, first, we analyze reliability in event estimation by defining a distortion metric. We characterize required spectrum opportunity to attain desired estimation distortion level while minimum amount of channels are accessed, based on spectrum mobility parameters, i.e., licensed user activity, interference, spectrum sensing and handoff durations, as well as real-time distributed sensing parameters, i.e., observation noise, delay-bound on reaching consensus, and energy consumption limitation per estimation interval. Then, we study required duration for reaching consensus among actor nodes after collection of samples from sensors and generating local estimate based on the interruption probability due to licensed user interference. Two regimes are identified: One in which sufficient spectrum opportunity exists and consensus can be achieved among actor nodes, and one in which actor nodes try to minimize the disagreement as much as they can support, i.e., provide opportunistic consensus, due to limited spectrum availability. The regime of operation depends on the licensed user activity on channels, and the early regime is desirable but achievable only for sparse licensed user existence on the spectrum. It is concluded that in certain environments under sparse spectrum occupancy by licensed users, spectrum access duration of sensor nodes can be adaptively reduced to enable convergence to consensus for actors in an estimation interval. Contribution of this paper can be outlined as follows

1. Analyze reliability in event detection: Local estimation distortion at actor node is modelled with respect to sensing signal-to-noise ratio (SNR), reporting rate and channel error rate.

2. Opportunistic Consensus: A cooperative scheme is proposed, where actor nodes share their local event estima- tions through opportunistic access of licensed channels to reach a global consensus about the event signal. We show that our scheme increases reliability.

3. Consensus Convergence: Impact of interruptions due to licensed user arrivals, mis-detection of licensed users, and wireless channel errors on reaching consensus is formulated. Prolongation in the consensus convergence time is studied under opportunistic spectrum access (OSA).

The remainder of the paper is organized as follows. In Section 2, distributed sensing architecture composed of cognitive radio sensor and actor nodes is elaborated. Problem formulation and reliability analysis is presented in Section 3. Factors that effect reliability are discussed in Section 4. Opportunistic consensus is analyzed in Section 5. Simulation results are presented in Section 6, and paper is concluded in Section 7.

\section{Network Setup}

We assume that the network in question consists of multiple wireless sensor nodes, each equipped with a cognitive radio (CR) and an actuator node with multiple CR transceivers. These nodes communicate among each other via opportunistic spectrum access (OSA) to perform remote monitoring and actuation tasks. Collected samples $s$ by sensor node $m$ on event signal $\theta$ are delivered to the actor node during the reporting interval $\tau_{r}$ over assigned channel $c$ within spectrum access duration $\tau_{a}$ to satisfy reliability requirement $D_{o}$ of distributed event observations. Reliability measure for estimation of the actor node is defined in terms of distortion $D$ (mean square-error) of the estimated event signal over a $\tau_{a}$.

Sensor nodes occupy a single CR transceiver with capability of adjusting its operating frequency to any channel $c$ in designated spectrum band by the actor node, i.e., channel set $C$ of the actor node. Channels are shared between selected sensor nodes participating in event data delivery. Sensor node behavior in a channel $c$ consists of data transmission $\tau_{t}$, spectrum sensing $\tau_{s}$ and spectrum handoff $\tau_{h}$ periods. We assume each sensor node is assigned to a specific channel for periodic spectrum sensing by the actor node, such that in spectrum sensing intervals $\tau_{s}$ all sensor nodes perform spectrum sensing to detect spectrum holes, i.e., vacant channels. With detection of licensed user, sensor node using that channel performs spectrum handoff and traverses channels provided by actor node to find a spectrum opportunity.

Spectrum handoff duration $\tau_{h}$ includes durations of consecutive channel switching $\tau_{\mathrm{cs}}$ and spectrum sensing $\tau_{s}$ at the new channel, until a vacant channel is found, i.e., duration between the instant where event reporting stopped due to licensed user detection and the instant when communication is resumed in a vacant channel. To achieve desired distortion level $D_{o}$ in an estimation interval $\tau_{e}$, sensors are assigned to channels by the actor node, such that they complete their transmission in spectrum access interval $\tau_{\mathrm{a}}$. Since $\tau_{e}$ includes $\tau_{\mathrm{a}}$ as well as delays due to cognitive cycle functionality durations, we have $\tau_{e}>\tau_{\mathrm{a}}$.

Channels assigned by the actor node, i.e., $c \in C$, are taken to be homogeneous in terms of licensed user activity and inter- 
ference. Energy constraints $\Omega$ for sensor nodes limit the energy that can be spent for event reporting in an estimation interval. These constraints are taken to be non-equal due to heterogeneous event arrivals in the field and remaining energy of sensor nodes. This energy variation brings limitation to event reporting duration $\tau_{r}$ of sensor nodes, and hence, causes heterogeneities for number of samples reported by each sensor.

\section{Reliable Distributed Sensing}

In this section, we lay out the problem formulation and present initial arguments of our analysis on reliability. We define a so called distortion metric $D$, which is the mean squared error (MSE) of our estimation of the event signal. We show how reliability depends on spectrum utilization of primary users (PU) as well as channel conditions.

Sensor nodes receive the event signal with varying power levels due to certain factors such as their distance from the event, device imperfections and noise. Furthermore, each sensor node has different amount of energy left in their battery. It is also possible that the sensor nodes utilize energy harvesting, in which case, the amount of energy harvested per unit time may vary from sensor to sensor. As a result, the amount of energy that can be consumed during the reporting of events $\Omega_{e}$, differ among the sensors.

There are also external influences on sensor event data reports such as varying wireless channel conditions, changes in PU channel utilization patterns, etc. Our aim is to incorporate all of these effects into a single metric that serves as a good indicator for reliability in event estimation.

We assume that the event signal at time $t$, i.e., $\theta(t)$, is received at sensor node $m$, with dispersion loss $\gamma(t)$ and added noise $\eta(t)$ as

$$
s_{m}(t)=\gamma_{m}(t) \cdot \theta(t)+\eta_{m}(t)
$$

For estimation of event signal from received samples from sensor nodes, actors use the best linear unbiased estimator (BLUE) [29] for local estimation. For a linear estimation type given above, where samples of $\eta_{m}$ are zero-mean, uncorrelated and have equal variances, BLUE is minimum-variance unbiased estimator. This allows us to make no assumptions on sensing noise distribution as long as noise power $\xi^{2}$ is known. Therefore, we do not assume any specific distribution for sensed signal and noise. Each sensor sends measured signal $s_{m}(t)$ to the actor node, where $\theta$ is estimated from the received version of $s_{m}(t)$.

We take both $\theta_{m}(t)$ and $\eta_{m}(t)$ are i.i.d. over time. We also assume that $\theta_{m}(t)$, and $\eta_{m}(t)$ have zero mean with power $\sigma_{\theta}^{2}$ and $\xi_{m}^{2}$, respectively. Sensors communicate with actor via opportunistic spectrum access. Actor generates an estimate of the event at the end of each event estimation interval. Under independence over time and space assumption, indices are ignored for all variables. This delay bounded distributed sensing scheme can also bee seen as real-time, since it put timeliness constraint on event reports. Received sample vector by the actor at the end of each event estimation interval $\tau_{e}$, i.e., $\mathbf{r}$, is combination of sensing noise and sensed signal as $\mathbf{r}=\boldsymbol{\gamma} \cdot \boldsymbol{\theta}+\boldsymbol{\eta}$, where $\mathbf{r}=\left[r_{1}, \cdots, r_{M_{s}}\right]^{T}, \gamma=\left[\gamma_{1}, \cdots, \gamma_{M_{s}}\right]^{T}$, and $\eta=\left[\eta_{1}, \cdots, \eta_{M_{s}}\right]^{T}$, where $M_{S}$ is the number of sensor nodes selected by actor node. Estimate of event signal using BLUE is formulated as follows [29]

$$
\hat{\theta}=\left[\boldsymbol{\gamma}^{T} \mathbf{R}_{\mathrm{s}}^{-1} \boldsymbol{\gamma}\right]^{-1} \boldsymbol{\gamma}^{T} \mathbf{R}_{\mathrm{s}}{ }^{-1} \mathbf{r}
$$

Sample covariance matrix $\mathbf{R}_{\mathbf{s}}$ for received samples at actor is a $M_{s}$ dimensional rectangular diagonal matrix whose diagonal entries are $\xi_{m}^{2}$, and other entries are 0 . We define a distortion metric $\tilde{D}$, as the the mean square error for BLUE can be determined as [29]

$$
\begin{aligned}
\tilde{D} & =E\left[(\hat{\theta}-\theta)^{2}\right]=\left[\boldsymbol{\gamma}^{T} \mathbf{R}_{\mathbf{s}}^{-1} \boldsymbol{\gamma}\right]^{-1} \\
& =\sum_{m=1}^{M_{s}}\left(\frac{\gamma_{m}^{2}}{\xi_{m}^{2}}\right)^{-1}
\end{aligned}
$$

Taking into account the fact that number of samples provided by each sensor $\zeta_{m}$, will be different due to various factors such as spectrum handoff and packet errors, we define a corresponding distortion metric $D$ as follows:

$$
D=\sum_{m=1}^{M_{s}}\left(\zeta_{m} \frac{\gamma_{m}^{2}}{\xi_{m}^{2}}\right)^{-1}
$$

Naturally, $\zeta_{m}$ depends on factors such as packet error rate $e_{m}^{(p)}$, and number of encountered spectrum handoffs. The number of packets that are correctly received by the actor can be expressed as

$$
\zeta_{m}=r_{m}^{(p)}\left(1-e_{m}^{(p)}\right)
$$

where, $r_{m}^{(p)}$ is the amount of packets that node $m$ was able to send within a reporting interval, while avoiding the PU by performing spectrum handoff whenever necessary. In the following section, we analyze the effects of these factors on $\zeta_{m}$, and consequently on distortion.

\section{Analysis of Factors that Effect Reliability}

In this section, we present an analysis of factors that effect reliability, such as spectrum handoff and packet errors.

\subsection{Impact of Spectrum Mobility}

Incorporation of cognitive radio capability into sensor and actor nodes enables reporting of event data over licensed bands to mitigate crowded spectrum problem. However, intermittent communications due to opportunistic spectrum access and interruptions due to licensed user interference must be addressed. To this end, actual spectrum access time of sensors, based on spectrum sensing and spectrum handoff functionalities is found.

Here, $r_{m}^{(p)}$, i.e., the experienced number of packets sent by node $m$ while utilizing licensed spectrum bands opportunistically is obtained. First, we derive the mean data communication duration $\mathrm{E}\left\{\tau_{\text {data }}\right\}$ before licensed user arrival in channels $C$ assigned by the actor node. Under exponential interarrival and inter-departure durations assumption for PU traffic $[23,24,25,26]$, average data communication duration $\mathrm{E}\left\{\tau_{\text {data }}\right\}$, 
i.e., average duration from starting communication in channel $C$ until a licensed user is detected is found as

$$
\mathrm{E}\left\{\tau_{\text {data }}\right\}=\mathrm{E}\{I\} \tau_{t}
$$

where $\mathrm{E}\{I\}$ is the mean number of $\left(\tau_{s}+\tau_{t}\right)$ durations before licensed user arrives at a channel in $C$. To calculate $\mathrm{E}\{I\}$, we define the probability of having $i$ intervals without licensed user arrival at the accessed channel as

$$
\operatorname{Pr}[I=i]=\left(P_{\text {hole }}\right)^{i-1}\left(1-P_{\text {hole }}\right)
$$

where $P_{\text {hole }}$ is vacancy probability of a channel in $C$. Mean of $I$ can be found as

$$
\mathrm{E}\{I\}=\sum_{i=1}^{\infty} i \cdot \operatorname{Pr}[I=i]=\frac{1}{1-P_{\text {hole }}} .
$$

To find the mean of spectrum handoff duration $\mathrm{E}\left\{\tau_{h}\right\}$, i.e., the mean of total channel switching and spectrum sensing durations until data transmission can be started in a vacant channel, we define probability of finding a vacant channel via $l$ consecutive handoffs $\operatorname{Pr}[L=l]$ as

$$
\operatorname{Pr}[L=l]=P_{\text {hole }}\left(1-P_{\text {hole }}\right)^{l-1}
$$

where we assume licensed user arrivals at different channels of $C$ are independent from each other. When a licensed user communication is detected at any of accessed channels sensor nodes accessing that channel traverses channels provided by the actor to find a vacant channel, where licensed user detected channel corresponds to $c=0$. Time spent while performing $l$ consecutive handoffs can be found as

$$
\tau_{h}(l)=l \cdot\left(\tau_{\mathrm{cs}}+\tau_{s}\right)
$$

where $\tau_{\mathrm{cs}}$ is channel switching time when moving between channels. As a result, $\mathrm{E}\left\{\tau_{h}\right\}$ can be obtained as

$$
\begin{aligned}
\mathrm{E}\left\{\tau_{h}\right\} & =\sum_{l=1}^{\infty} \tau_{h}(l) \operatorname{Pr}[L=l] \\
& =\sum_{l=1}^{\infty} l \cdot\left(\tau_{\mathrm{cs}}+\tau_{s}\right) P_{\text {hole }}\left(1-P_{\text {hole }}\right)^{l-1} \\
& =\left(\tau_{\mathrm{cs}}+\tau_{s}\right) \frac{1}{P_{\text {hole }}}
\end{aligned}
$$

To find the number of packets sent by sensor node $m$, i.e., $r_{m}^{(p)}$, we use the fact that the total time spent by a node in spectrum sensing, data transmission and spectrum handoff must not exceed the reporting period $\tau_{r}$. With this restriction, we can find the expected number of handoffs experienced by sensor $m$, i.e., $h_{m}$. We require

$$
E\{I\}\left(\tau_{s}+\tau_{t}\right)+h_{m}\left(E\left\{\tau_{h}\right\}+E\{I\}\left(\tau_{s}+\tau_{t}\right)\right)
$$

Therefore, we need the maximum value of $h_{m}$ that satisfies

$$
h_{m} \leq \frac{\tau_{r}-E\{I\}\left(\tau_{s}+\tau_{t}\right)}{E\left\{\tau_{h}\right\}+E\{I\}\left(\tau_{s}+\tau_{t}\right)}
$$

If we define such $h_{m}$ as $h_{m}^{*}$,

$$
r_{m}^{(p)}=\frac{h_{m}^{*} E\left\{\tau_{\text {data }}\right\} v_{m}}{8 l_{p}}
$$

where $l_{p}$ is the packet size in bytes and $v_{m}$ is the transmission rate in bits/sec. Instantanepus throughput can be defined as $T=$ $r_{m}^{(p)} / \tau_{r}$

\subsection{Packet Error Rate}

Each sensor communicates with the actor node over orthogonal channels that experience independent shadowing and zeromean AWGN. We use the log-normal channel for received power calculations, which is experimentally shown as the accurate model for low power communication in sensor networks [27]. In this model, the received power at a receiver at distance $d$ from a transmitter is given by

$$
P_{r}(d)=P_{t}-\operatorname{PL}\left(d_{0}\right)-10 \eta \log _{10}\left(\frac{d}{d_{0}}\right)+X_{\sigma},
$$

where $P_{t}$ is the transmit power in $\mathrm{dBm}, \operatorname{PL}\left(d_{0}\right)$ is the path loss at the reference distance $d_{0}$ in $\mathrm{dB}, \eta$ is the path loss exponent, and $X_{\sigma}$ is the shadow fading component with $X_{\sigma} \sim \mathcal{N}(0, \sigma)$. We denote the communication signal-to-noise ratio (SNR) at the actor with and without licensed user interference as $\Psi_{w}$, and $\Psi_{l}$, respectively. SNR at the receiver without licensed user interference $\Psi_{w}$ is given by $\Psi_{w}=P_{r}(d)-P_{n}$ in $\mathrm{dB}$, where $P_{n}$ is the communication noise power in $\mathrm{dBm}$. On the other hand, SINR at the receiver due to licensed user activity is given by $\Psi_{l}=P_{r}(d)-P_{n}-P_{l}^{(k)}$ in $\mathrm{dB}$, where $P_{l}^{(k)}$ is the interference caused by licensed user activity at actor node $k$. To obtain bit error rate $P_{b}$ to use in analytical derivations, the non-coherent frequency shift keying (FSK) modulation scheme is selected. The bit error rate of this scheme is given by [28]

$$
P_{b}=\frac{1}{2} \exp \left(-\left(E_{b} / N_{0}\right) / 2\right), \quad E_{b} / N_{0}=\Psi \frac{B_{N}}{R}
$$

where $B_{N}$ is the noise bandwidth, and $R$ is the data rate. Finally, packet error rate $\left(P_{p}\right)$ for packet length $l_{p}$ becomes

$$
P_{p}=1-\left(1-P_{b}\right)^{l_{p}}
$$

To determine average packet error rate $e_{m}^{(p)}$ incorporating licensed user interference due to opportunistic spectrum access, we propose an error model based on false-alarm $P_{f}$ and detection $P_{d}$ probabilities, and licensed user state transition, i.e., birth $\beta$ and death $\alpha$ rates. False-alarm, i.e., detection of licensed user communication when licensed user is actually not communicating, probability is represented by $P_{f}$ and detection probability when licensed user is communicating $P_{d}$. Licensed user activity modelled using two state discrete Markov chain model with ON and OFF states [23, 24, 25, 26]. Licensed user being active $\left(1-P_{\text {hole }}\right)$ and being inactive $P_{\text {hole }}$ probabilities are equal to $\beta /(\beta+\alpha)$ and $\alpha /(\beta+\alpha)$, respectively. Before finding $e_{m}^{(p)}$, we describe two different cases in which licensed user interference occurs as follows 


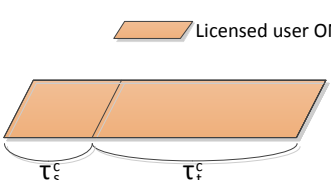

(a)

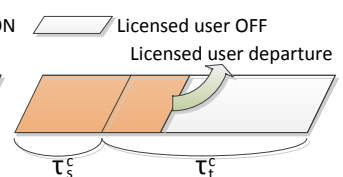

(b)
Figure 1: Licensed user interference patterns when mis-detection occurs, no state change in (a) and state transition occurs in (b).

1. Although licensed user is active, it may not be detected, i.e., mis-detection can occur, with probability (1 $\left.P_{\text {hole }}\right)\left(1-P_{d}\right)$. In this case, nodes continue to communicate, though there is ongoing licensed user communication. Hence, they get exposed to licensed user interference, which is illustrated in Fig. 1.

2. A spectrum band can be identified as vacant and employed by sensor nodes with probability $P_{\text {hole }}\left(1-P_{f}\right)$. However, licensed users may start communication during the nodes' transmission period. Thereby, until the next spectrum sensing period, sensor nodes are unaware of the licensed user presence and continue to communicate under licensed user interference, which is illustrated in Fig. 2.

Licensed user may change its state during $\tau_{t}$, or it can keep its state along whole $\tau_{t}$ duration. Having $\tau_{t}$ is relatively small with respect to average active and inactive durations of licensed user, e.g., $1 / \alpha$ and $1 / \beta$, respectively, is a more realistic assumption than having large $\tau_{t}$ that is longer than licensed user transmission duration. Thus, we assume $\tau_{t}$ is smaller than both $1 / \alpha$ and $1 / \beta$.

Interference due to mis-detection exhibits two different patterns according to $\tau_{t}$ as in Fig. 1 (a) and (b). It can be through whole $\tau_{t}$ period as in Fig. 1 (a) or licensed user state may change to inactive from active as in Fig. 1 (b). The probability that the licensed user is transmitting during the entire $\tau_{t}$ can be obtained as $e^{-\alpha \tau_{t}}$, and the probability that licensed user goes inactive state from active during $\tau_{t}$ can be found as $1-e^{-\alpha \tau_{t}}$. If the licensed user state does not change during the $\tau_{t}$, the licensed user interference persists over the entire transmission period and in which error rate is $P_{p}^{l}$. However, if state transition between active and inactive states occurs during $\tau_{t}$, average error rate converges to $\left(1-P_{\text {hole }}\right) P_{p}^{l}+P_{\text {hole }} P_{p}^{w}$. Thus, average packet error rate for the mis-detection case $P_{p}^{1}$ can be expressed as

$$
\begin{aligned}
P_{p}^{1} & =\left(1-P_{d}\right) \frac{\beta}{\alpha+\beta} \\
& \cdot\left(\left(1-e^{-\alpha \tau_{t}}\right) \frac{\beta P_{p}^{l}+\alpha P_{p}^{w}}{\alpha+\beta}+e^{-\alpha \tau_{t}} P_{p}^{l}\right)
\end{aligned}
$$

Similarly, if licensed user is inactive and there is no falsealarm, interference only happens when licensed user state transition occurs during $\tau_{t}$, as illustrated in Fig. 2 (b). Average packet error rate converges to again approximately to

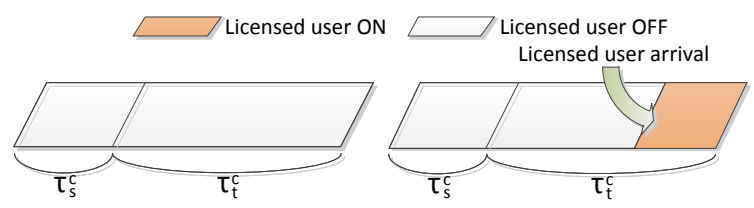

(a)

(b)

Figure 2: Licensed user interference patterns when licensed user is initially OFF while spectrum sensing, no state change in (a) and state transition occurs in (b).

$\left(1-P_{\text {hole }}\right) P_{p}^{l}+P_{\text {hole }} P_{p}^{w}$ with probability $1-e^{-\beta \tau_{t}}$. Average packet error rate in this case $P_{p}^{2}$ becomes

$$
\begin{aligned}
P_{p}^{2} & =\left(1-P_{f}\right) \frac{\alpha}{\alpha+\beta} \\
& \cdot\left(\left(1-e^{-\beta \tau_{t}}\right) \frac{\beta P_{p}^{l}+\alpha P_{p}^{w}}{\alpha+\beta}+e^{-\beta \tau_{t}} P_{p}^{w}\right)
\end{aligned}
$$

Then, to find overall packet error rate $e_{m}^{(p)}$ in opportunistic spectrum access incorporating licensed user interference, we add and normalize $P_{p}^{1}$ and $P_{p}^{2}$ as

$$
e_{m}^{(p)}=\frac{P_{p}^{1}+P_{p}^{2}}{\frac{\beta\left(1-P_{d}\right)+\alpha\left(1-P_{f}\right)}{\alpha+\beta}}
$$

\subsection{Spectrum Access Duration and Sensor Scheduling Order}

The analysis presented in the previous sections provides us with the following expression for distortion metric

$$
\begin{aligned}
D & =\sum_{m=1}^{M_{s}}\left(\zeta_{m} \frac{\gamma_{m}^{2}}{\xi_{m}^{2}}\right)^{-1} \\
& =\sum_{m=1}^{M_{s}}\left(\left(1-e_{m}^{(p)}\right) r_{m}^{(p)} \frac{\gamma_{m}^{2}}{\xi_{m}^{2}}\right)^{-1} \\
& =\sigma_{\theta}^{2} \sum_{m=1}^{M_{s}}\left(\left(1-e_{m}^{(p)}\right) r_{m}^{(p)} \Lambda_{m}\right)^{-1} \\
& =\frac{\sigma_{\theta}^{2}}{8 l_{p} E\left\{\tau_{\text {data }}\right\}} \sum_{m=1}^{M_{s}}\left(h_{m}^{*} \nu_{m}\left(1-e_{m}^{(p)}\right) \Lambda_{m}\right)^{-1}
\end{aligned}
$$

Minimum number of sensor nodes satisfying distortion constraint can be reached via ordering sensors in the descending sensing SNR $\left(\Lambda_{m}\right)$ order, i.e., $m=1$ is the largest SNR sensor node, and using in (3) with maximum allowed $\tau_{r}^{(m)}$ until distortion constraint is satisfied. According to this formulation, instead of using all sensors, only the right group of sensor nodes can be determined and still the distortion threshold $D_{0}$ can be achieved.

In this section, we analyze the contribution of data carried at each channel to distortion. The event observation distortion at an actor node is discretized into contribution of each 
channel $D_{c}$, i.e., total distortion is expressed as the addition of samples belonging to different channels, addressing the reduction in the reported samples over a channel by the spectrum access duration $\tau_{a}$ during estimation interval $\tau_{e}$. Sensor nodes can move among spectrum holes via spectrum handoff as a licensed user arrives at current accessed channel. However, sensor nodes' communication is interrupted by periodic spectrum sensing as well as spectrum handoff triggered by licensed user arrival. Therefore, effect of opportunistic spectrum availability on event sensing reliability is investigated analytically according to proposed distributed sensing distortion model.

To this end, we define $\mathcal{M}^{(c)}$ as the source sensors of the actor assigned to $c^{\text {th }}$ channel for sharing during $\tau_{a} . D$ for proposed distributed sensing scheme can be expressed as

$$
D=\left(\sum_{c} D_{c}^{-1}\right)^{-1}
$$

where $D_{c}$ is the distortion resulted by observations of sensors at channel $c$. It is dependent on the accessible duration $\tau_{a}$ of a channel assigned by the actor during an estimation interval and required reporting duration of sensors $\tau_{r}^{(m)}$ sharing the same channel. $\tau_{a}$ s are independent and identically distributed (i.i.d.) random variables with mean $\mu$ representing data communication duration in an estimation interval for channels of the actor node. $D_{c}$ is the distortion achieved when spectrum access duration $\tau_{a}$ in an estimation interval is allowing $\omega^{\text {th }}$ sensor starting from $m=1$ to share that channel for event reporting, and it can be expressed as

$$
\begin{aligned}
& D_{c}=\left[\sum_{m=1}^{\omega-1} D_{m}^{-1}+D_{\omega}^{-1}\left(\frac{\tau_{a}-\sum_{m=1}^{\omega-1} \tau_{r}^{(m)}}{\tau_{r}^{(\omega)}}\right)\right]^{-1}, \\
& \text { for } \sum_{m=1}^{\omega-1} \tau_{r}^{(m)} \leq \tau_{a} \leq \sum_{m=1}^{\omega} \tau_{r}^{(m)}, m \in \mathcal{M}, \omega \in \mathcal{M}
\end{aligned}
$$

where $\omega$ is the last sensor node that was reporting using the channel $c$ at the instant of licensed user arrival, and $D_{m}$ is distortion solely depending on samples from sensor $m$, which can be obtained using formulation in Section 4.3 as

$$
\frac{\sigma_{\theta}^{2}}{8 l_{p} E\left\{\tau_{\text {data }}\right\}}\left(h_{m}^{*} v_{m}\left(1-e_{m}^{(p)}\right) \Lambda_{m}\right)^{-1}
$$

Furthermore, it is deduced that $D_{c}$ is equal to $\left(\sum_{m=1}^{\left|\mathcal{M}^{(c)}\right|} D_{m}^{-1}\right)^{-1}$ for $\tau_{a} \geq \sum_{m=1}^{\left|\mathcal{M}^{(c)}\right|} \tau_{r}^{(m)}$. When $\tau_{a} \geq \sum_{m=1}^{\left|\mathcal{M}^{(c)}\right|} \tau_{r}^{(m)}$, sensors assigned to channel $c$ are able to find an opportunity to completely transmit their observations to actor during the estimation interval, and desired $D_{c}$ will be achieved. However, when $\tau_{a} \leq \mu$, reporting of sensors assigned to a channel are limited by spectrum access duration $\left(\tau_{a} \leq \sum_{m=1}^{\left|\mathcal{M}^{(c)}\right|} \tau_{r}^{(m)}\right)$, and contribution to total estimation distortion $D_{c}$ is calculated using (23) via replacing $\tau_{a}$ with its realization. Latest reporting sensor $\omega$ at accessed channels can be determined via $\sum_{m=1}^{\omega-1} \tau_{r}^{(m)} \leq \tau_{a} \leq \sum_{m=1}^{\omega} \tau_{r}^{(m)}$, and its unfinished reporting duration can be found as $\tau_{a}-\sum_{m=1}^{\omega-1} \tau_{r}^{(m)}$.
Each channel's contribution to distortion of total estimation at actor varies based on sensing SNR $\Lambda_{m}$ of assigned sensors to a channel. Therefore, another important issue is the scheduling order of source nodes assigned to same channel. Addressing the interruptions due to opportunistic communication, and hence, random $\tau_{a}$ duration in an estimation interval $\tau_{e}$, scheduling spectrum access of source nodes with respect to their observation SNR $\Lambda_{m}$ in descending order, i.e., highest SNR having node $(m=1)$ accesses first at designated channel, is found favourable.

\section{Opportunistic Consensus}

In this section, based on the previous analysis, we propose a consensus scheme to increase reliability of event estimation. We assume a case where there are multiple actor nodes, each with its own set of sensors. Each sensor-actor group of nodes operate as described in previous sections. However, after actor nodes make a local estimation of the event signal, they communicate their local estimate with other actors to mitigate disagreement and reach a global agreed state. We, first, present an overview of used consensus model, and then, investigate consensus convergence duration with respect to different algebraic connectivity of actor network for various licensed user arrival $\beta$ and interference $P_{l}$ values.

While reaching a consensus is trivial for small scale actor networks, for a large scale actor network computing the global estimate $\Theta$ based on local estimates $\hat{\theta}_{k}$ requires multiple iterations and packet exchanges among actors. Inter-actor communication is subject to licensed user interference. This can either be in the form of licensed user arrival during transmission or mis-detection of licensed user presence during spectrum sensing period. In case of transmission errors, iteration is stopped and this failed step is repeated via automatic repeat request. The exchange of information among actors is performed via a slotted and collision-free medium access control (MAC) scheme. Consensus convergence time $\tau_{c}$ is determined as the multiplication of number of required iterations $\Upsilon$ to reach consensus and iteration duration $\tau_{i}$, i.e., $\tau_{c}=\Upsilon \cdot \tau_{i}$.

\subsection{Overview of Consensus Model}

We use a cognitive radio actor network composed of $K$ actor nodes. Actor $k$ can communicate with actor $l$ if $l$ is a neighbor of $k$ on a graph $G=(V, E)$, where $V$ denotes the set of vertices, i.e., actor nodes, and $E \subseteq V \times V$ denotes set of the edges between vertices, i.e., links between actor nodes. The adjacency matrix A of the graph $G$ is composed of binary-valued entries $a_{k, l}$ that indicates existence of a link between actor $k$ and $l$. Value of $a_{k, l}$ is equal to 1 if actor $k$ and $l$ are connected, and $a_{k, l}=0$ if there is no link between actor $k$ and $l$. We assume the links $E$ are bidirectional, and hence, the graph $G$ is undirected. Furthermore, $\mathbf{A}$ is a symmetric matrix with 0 diagonal entries, and since $\sum_{k \neq l} a_{k, l}=\sum_{l \neq k} a_{l, k}$, the undirected graph $G$ is called balanced. The degree matrix $\mathbf{D}$ of $G$ is a diagonal matrix with entries $d_{k, k}=\left|\mathcal{N}_{k}\right|$ and zero off-diagonal elements, where $\left|\mathcal{N}_{k}\right|=\sum_{k \neq l} a_{k, l}$ is the number of nodes in the neighborhood set 
$\mathcal{N}_{k}$ of actor $k$. Let $\mathbf{L}$ be the Laplacian matrix of the graph $G$, which is defined as $\mathbf{L}:=\mathbf{D}-\mathbf{A}$. Entries $l_{k, l}$ of $\mathbf{L}$ are as

$$
l_{k, l}=l_{l, k}= \begin{cases}\left|\mathcal{N}_{k}\right| & \text { if } k=l \\ -a_{k, l} & \text { if } k \neq l\end{cases}
$$

Aim of consensus algorithm is to make each actor node reach a globally optimal estimate $\hat{\theta}^{*}$ from a set of measurements, i.e., local estimates $\hat{\theta}_{k}$ of actor nodes in our system model. This is achieved via exchange of local estimates among nearby actors without utilization of a fusion center. By definition of $\mathbf{L}$ sum of all columns or rows is equal to $\mathbf{0}$, which implies that $\mathbf{L}$ has an eigenvalue of 0 . Accordingly, associated eigenvector is $\mathbf{1}$ since $\mathbf{L 1}=\mathbf{0}$, or $\mathbf{1}^{\mathrm{T}} \mathbf{L}=\mathbf{0}^{\mathrm{T}}$. Therefore, reached final estimate is of the form $\alpha \mathbf{1}$, and consensus value $\alpha$ is equal to average of local estimates of actors. If $G$ is a connected balanced and undirected graph, a consensus is asymptotically reached with

$$
\Theta=\sum_{k} \hat{\Theta}_{k}(0) / K
$$

Since minimization of the difference between states of actors is the aim of consensus algorithm, the disagreement, i.e., error, function is defined as

$$
J(\hat{\Theta})=\frac{1}{2} \hat{\boldsymbol{\Theta}}^{\mathrm{T}} \mathbf{L} \hat{\boldsymbol{\Theta}}=\frac{1}{4} \sum_{k=1}^{K} \sum_{l=1}^{L} a_{k, l}\left(\hat{\Theta}_{k}-\hat{\Theta}_{l}\right)^{2}
$$

Using steepest descent technique the minimum of $J(\Theta)$ can be achieved in discrete time as

$$
\hat{\Theta}_{k}(t+1)=\hat{\Theta}_{k}(t)+\vartheta \sum_{l \in \mathcal{N}_{k}} a_{k, l}\left(\hat{\Theta}_{k}(t)-\hat{\Theta}_{l}(t)\right)
$$

where $\vartheta$ is the step-size. In matrix form, dynamics of the actor network can be expressed as

$$
\hat{\boldsymbol{\Theta}}(t+1)=\mathbf{P} \hat{\boldsymbol{\Theta}}(t)
$$

where $\mathbf{I}$ is the identity matrix, and $\mathbf{P}=\mathbf{I}-\vartheta \mathbf{L}$. Value of $\vartheta$ is selected appropriately to ensure all eigenvalues of $\mathbf{P}$ are less than 1. The consensus algorithm is initialized with local estimates of actor nodes, i.e., $\hat{\Theta}_{k}(0)=\hat{\theta}_{k}$.

In the next section, the described inter-actor distributed estimation framework is used for assessing consensus convergence duration under opportunistic spectrum access.

\subsection{Algebraic Connectivity and Consensus Convergence}

While the performance of consensus algorithm is characterized by its convergence rate, i.e., time elapsed to reach consensus, convergence speed is lower bounded by the lowest nonzero eigenvalue of $\mathbf{P}$, i.e., $\lambda_{2}$, which is also called algebraic connectivity of the graph. Consensus is reached exponentially in discrete-time for connected graphs [5] such that

$$
\frac{\|\hat{\boldsymbol{\Theta}}(t)-\boldsymbol{\Theta}\|}{\|\hat{\boldsymbol{\Theta}}(0)-\boldsymbol{\Theta}\|} \leq \mathrm{O}\left(e^{-\left(1-\vartheta \lambda_{2}(\mathbf{L})\right) \tau_{c}}\right)
$$

We state the reaching consensus condition as $\frac{\left\|\boldsymbol{\theta}(t)-\boldsymbol{\theta}^{*}\right\|}{\left\|\boldsymbol{\theta}(0)-\boldsymbol{\theta}^{*}\right\|} \leq \varepsilon$. Therefore, the time elapses until convergence $\tau_{c}$ can be defined as the time needed to smallest eigenvalue of the dynamical system can be reduced by a factor $\varepsilon \ll 1$. A step of iteration fails if licensed user arrives or in case of mis-detection during communication of local estimate. Then, a new vacant channel is moved and failed step is repeated there. Failed step is detected after the end of iteration, hence, iteration is re-started. Instead of trying to know when exactly pu arrived during iteration. To mitigate disagreement for a unit amount $1 /\left(1-P_{p}\right) \cdot 1 /\left(1-\vartheta \lambda_{2}(\mathbf{L})\right)$ iterations are needed, and hence, $\tau_{c}$ is found as

$$
\tau_{\mathrm{c}}=\frac{1}{1-P_{p}} \frac{\log (\varepsilon)}{1-\vartheta \lambda_{2}(\mathbf{L})} \mathrm{E}\left\{\tau_{i}\right\}
$$

where $\tau_{i}$ is the time required for a single iteration, i.e., duration in which all actor nodes communicate their local state once with their neighbor actors. We elaborate calculation of mean of $\tau_{i}$ in the following.

\subsection{Estimation Interval Partitioning For Consensus}

Estimation interval must be partitioned adaptively addressing varying spectrum parameters with spectrum mobility. Objective of our scheme is to provide sufficient duration for communication of local states among actor nodes after completion of local estimation. We first describe MAC for inter-actor communication and formulate mean iteration duration $\mathrm{E}\{i\}$. Then, estimation interval partitioning is introduced.

Actor nodes employ a $\rho$-persistent carrier sense multiple access (CSMA) mechanism for MAC such that they access channel during slot intervals with a probability of $\rho$ if channel is no other actor node is sensed to be transmitting. Expected number of actor nodes that will transmit when channel becomes idle is given by $\left|\mathcal{N}_{k}\right| \rho$. If $\left|\mathcal{N}_{k}\right| \rho>1$, then a collision is expected to occur. To prevent collisions and resultant retransmissions, $\rho$ should be chosen accordingly, i.e., $\rho<1 /\left|\mathcal{N}_{k}\right|$. For this scheme, successful transmission probability of actor node $k$ can be found as

$$
\zeta_{k}=\rho(1-\rho)^{\left|\mathcal{N}_{k}\right|-1}
$$

Using $\varsigma_{k}$, mean number of channel access trials $\varphi_{k}$ before gaining access to channel is found as

$$
\mathrm{E}\left\{\varphi_{k}\right\}=\sum_{l=0}^{\infty} l \varsigma_{k}\left(1-\varsigma_{k}\right)^{l-1}
$$

Finally, mean duration of an iteration can be found as

$$
\mathrm{E}\left\{\tau_{i}\right\}=\sum_{k=1}^{K} \tau_{\text {slot }} \mathrm{E}\left\{\varphi_{k}\right\}
$$

where $\tau_{\text {slot }}$ is the channel access duration of an actor node while broadcasting its local state to neighbor actors. Found $\mathrm{E}\left\{\tau_{i}\right\}$ is incorporated into (31), to find $\tau_{c}$.

Spectrum access duration for sensor nodes is adjusted to allow convergence of consensus algorithm. To this end, although, higher available spectrum access $\tau_{a}$ duration is possible, $\tau_{a}$ is reduced at the expense of increased number of required channels during local estimation. Reduced spectrum access duration $\tau_{a}^{*}$ is determined as

$$
\tau_{a}^{*}=\min _{k}\left\{\mu_{k}\right\}-\tau_{c}
$$




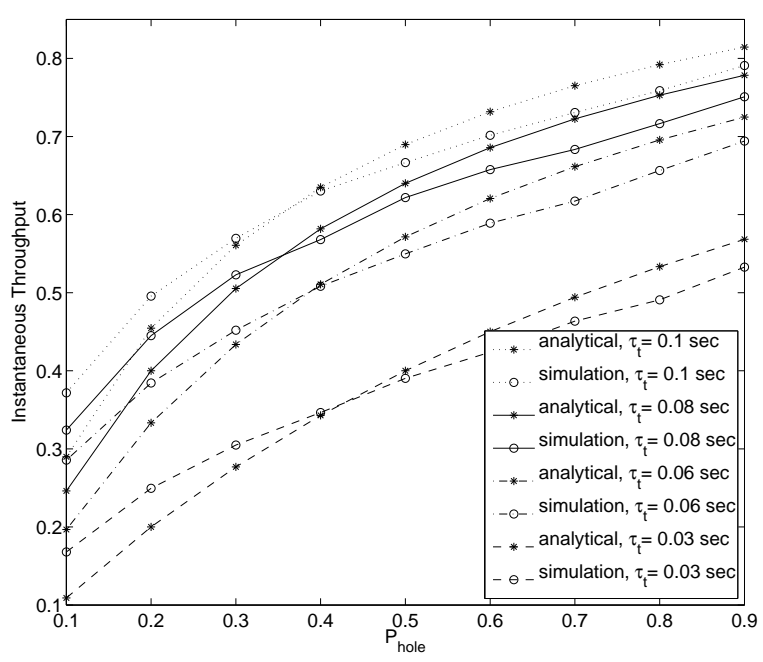

Figure 3: Comparision of calculated and achieved instantaneous throughput due to opportunistic spectrum access for $\tau_{t}=0.1, \tau_{t}=0.08, \tau_{t}=0.06, \tau_{t}=0.03$ sec.

where $\tau_{a}^{*} \geq \max _{m} \tau_{r}^{(m)}$. Using developed scheme, $\alpha$ and $\beta$ values are found. Instantaneous throughput values, $T_{k}$, for channels $C_{k}$ of actor node $k$ under opportunistic spectrum access are determined and updated according to spectrum opportunities. We now provide performance assessment of our scheme.

\section{Performance Evaluation}

In this section, we provide simulation results related to the provided analysis and the proposed consensus scheme.

\subsection{Instantaneous Throughput}

Performance of proposed instantaneous throughput determination model is shown in Fig. 3. Analytically calculated instantaneous throughput is shown to be closely following simulations. $\tau_{s}$ and $\tau_{\mathrm{cs}}$ is set to 0.02 and $0.005 \mathrm{sec}$, respectively. $P_{\text {hole }}$ is varied from 0.1 to 0.9 . Calculated instantaneous throughputs for $\tau_{t}=0.1,0.08,0.06$, and $0.03 \mathrm{sec}$ overshoot the achieved ones after a critical value of $P_{\text {hole }}$, e.g., after $P_{\text {hole }}=0.3$ calculated instantaneous throughput exceeds the achieved one for $\tau_{t}=0.08 \mathrm{sec}$., and similar relationship between simulated and analytic results is observed for other $\tau_{t}$ values, as well. Therefore, in our design of consensus algorithm, we introduce safety factor $\kappa$ to reduce calculated throughput by a little amount to compensate for possible overshoots.

We use developed framework to evaluate the instantaneous throughput values, $T_{k}$, and calculate the mean of the spectrum access duration $\mu_{k}$ in an event estimation interval for sensor nodes connected to actor node $k$, i.e., $m \in \mathcal{M}_{k}$, as $\mu_{k}=T_{k} \tau_{e}$.

\subsection{Error Rate}

Accuracy of proposed analytical packet error rate calculation scheme is presented in Fig. 4. For received power calculations,

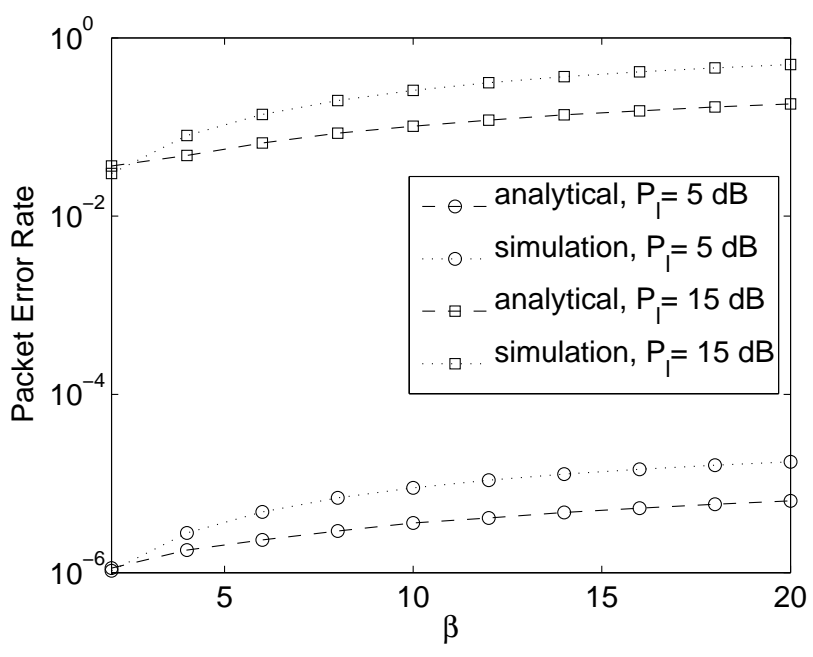

Figure 4: Comparison of analytically calculated packet error rate and simulation results with respect to licensed user arrival rate $\beta$ for SNR values under licensed user interference of $P_{l}=5$ and $15 \mathrm{~dB}$.

transmission power $P_{t}$ is set to $-5 \mathrm{dBm}$, noise power $P_{n}$ is set to $-90 \mathrm{dBm}$, path loss exponent $\eta$ is set to 3 , shadowing standard deviance $\sigma$ is set to 3.8 , distance is taken to be $5 \mathrm{~m}$, reference distance $d_{0}$ is taken to be $1 \mathrm{~m}$, reference path loss $P L\left(d_{0}\right)$ is taken as $-40 \mathrm{~dB}$, and $B_{N} / R$ ratio is taken to be unity. Value of $\tau_{t}$ is taken to be $0.05 \mathrm{sec}, P_{d}$ is equal to $0.9, P_{f}$ is equal to 0.1 , and $\alpha$ is equal to 10. Simulations are repeated 1000 times and results are averaged. It is shown in Fig. 4, the analytic formulation follows the simulation values closely. While packet error rate is in the order of $10^{-5}$ when the licensed user interference $P_{l}$ is $5 \mathrm{~dB}$, packet error rate becomes in the order of $10^{-2}$ when $P_{l}$ increases to $15 \mathrm{~dB}$.

\subsection{Consensus Convergence Duration}

Numerical results are provided to display the consensus convergence duration with respect to algebraic connectivity $\lambda_{2}(\mathbf{L})$ and available spectrum opportunity in terms of licensed user interference and channels errors. The prolongation of consensus convergence duration with respect to various algebraic connectivity values of actor graph $G$, i.e., from 1 to 20 , is discussed. Value of $\varepsilon$ is set to $-40 \mathrm{~dB}$. Value of $\mathrm{E}\left\{\tau_{i}\right\}$ is set to $0.05 \mathrm{sec}$. To investigate effect of spectrum mobility, effect of licensed user interference $P_{l}$ is assessed for 5 and $10 \mathrm{~dB}$, and effect of birth rate of licensed user $\beta$ is assessed for 10 and 20 .

In Fig. 5, we show that as the instantaneous throughput decreases, required number of channels to schedule sensors increases. However, this decrease becomes marginal after a certain point, e.g., $T_{k}=0.8$. This fact also suggests that it is possible to decrease spectrum access duration for sensor-to-actor communication, $\tau_{a}$, to provide sufficient time opportunity to reach consensus among actor nodes without increasing spectrum efficiency by increasing required number of channels under sparse licensed user activity.

For consensus convergence duration $\tau_{c}$, it has been shown in Fig. 6 that as the algebraic connectivity $\lambda_{2}$ increases, effect of 


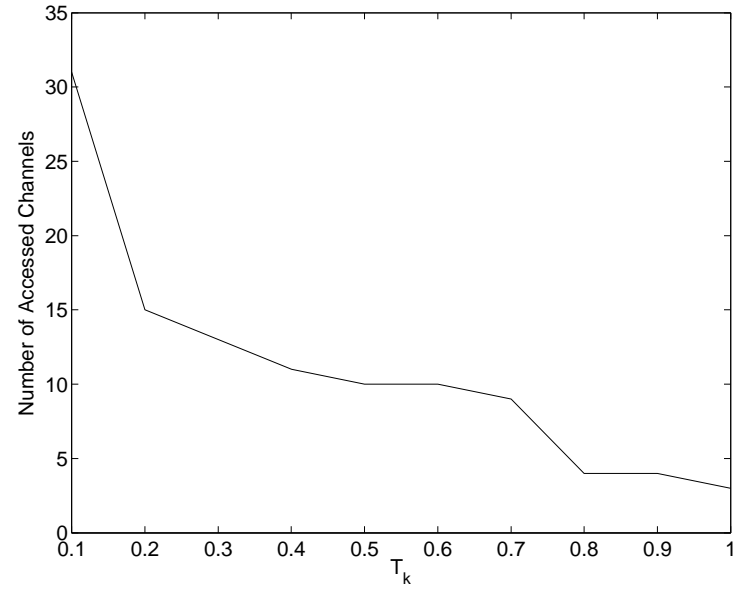

Figure 5: Required number of channels with respect to instantaneous throughput $T_{k}$ for $\tau_{e}=1 \mathrm{sec}$ and distortion constraint $D_{o}$ of $-50 \mathrm{~dB}$.

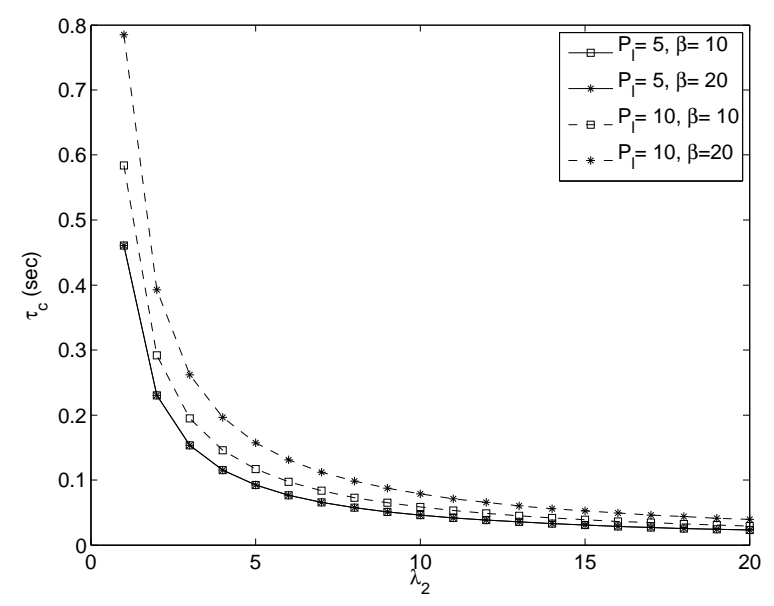

Figure 6: Consensus duration with respect to algebraic connectivity for various $P_{l}$ and $\beta$ values.

spectrum mobility, i.e., $P_{l}$ and $\beta$, decreases. Effect of increasing $\lambda_{2}$ becomes marginal after a certain point, e.g., $\tau_{c}$ reduces to its half value when $\lambda_{2}$ increases from 1 to 2 , however, when $\lambda_{2}$ is increased from 9 to 10 , this increase does not imply any significant reduction of $\tau_{c}$. For $P_{l}=5 \mathrm{~dB}, \tau_{c}$ follows the same pattern for $\beta=10$ and 20. It is deduced that for low licensed user interference, $\beta$ value does not effect the $\tau_{c}$. However, for $P_{l}=10 \mathrm{~dB}$, experienced $\tau_{c}$ increases with $\beta$. Sufficient time opportunity to reach consensus among actor nodes should be provided to reach consensus before end of the estimation interval. Therefore, we incorporate analytically formulated consensus duration to limit spectrum access duration for local estimation of actors, i.e., $\tau_{a}$.

\subsection{Adaptive Spectrum Sharing for Consensus}

In this section, we present simulation results on the performance of proposed sensing framework. In Fig. 7, consensus reaching performance is presented with respect to algebraic

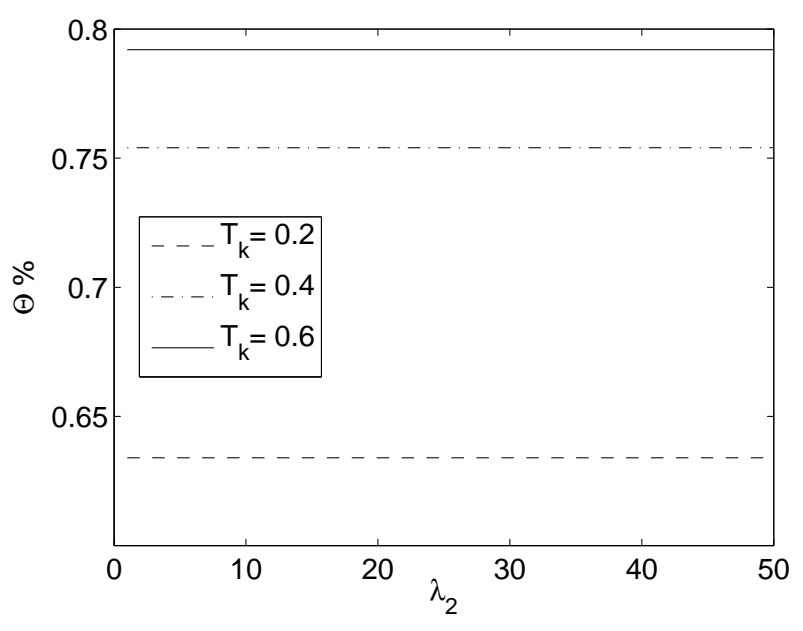

Figure 7: Consensus reaching rate of actor nodes.

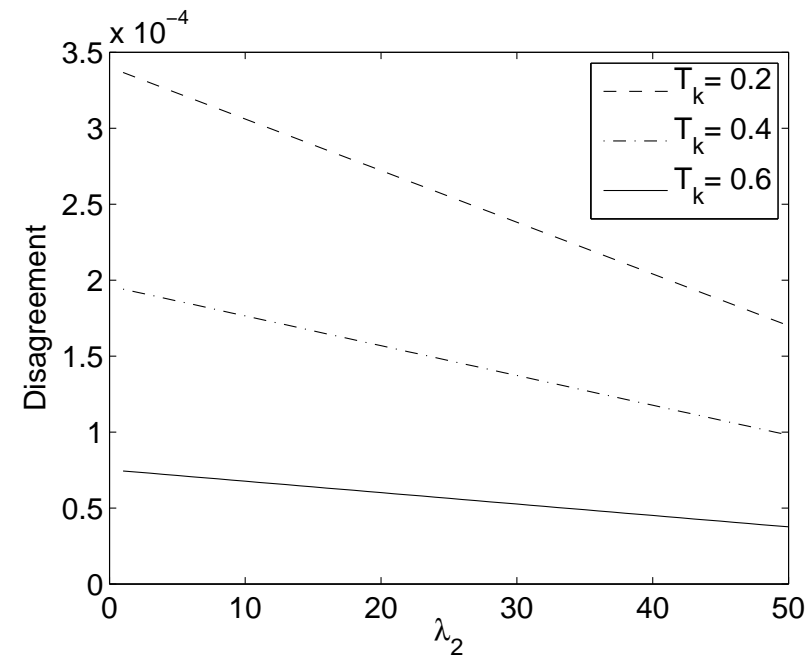

Figure 8: Consensus disagreement of actor nodes.

connectivity for different $T_{k}$ values. $P_{l}$ is set to $15 \mathrm{~dB}$. It is shown that for different $\lambda_{2}$ values, consensus is reached irrespective of algebraic connectivity. Our scheme achieves higher consensus reaching rate performance with high instantaneous throughput values, i.e., consensus reaching rate decreases with decreasing $\min \left\{T_{k}\right\}=$ from 0.6 to 0.2 . Reducing spectrum access duration of sensor nodes in local estimation, provides this performance gain in channels having high licensed user activity spectrum bands. Proposed estimation interval partitioning scheme strictly reduces spectrum access interval in accordance with sparse spectrum availability and high licensed user interference. Reduction of local estimation duration is benefited in high licensed user interference scenarios, and for low instantaneous throughput case, higher duration for consensus convergence is left.

In Fig. 8, disagreement among actors at the end of estimation interval is presented. With increasing algebraic connectivity $\lambda_{2}$, 
left duration for consensus decreases. Therefore, higher number of incomplete consensus phases yields higher disagreement than desired disagreement $\varepsilon$, which is set to $10^{-4}$. Since the predicted $\tau_{c}$ decreases with increased $\lambda_{2}$, spectrum access interval for local estimation increases, as well. Due to randomness in licensed user activity, this reduction in $\tau_{c}$ results in increase of disagreement among actor nodes. However, as it is shown in Fig. 8, disagreement is still very close to and in the order of the objective value.

\section{Conclusions}

In this paper, reliable spectrum access and reaching consensus with cognitive radio sensor and actor nodes is discussed. Furthermore, a consensus scheme is proposed to increase reliability by enabling consensus convergence of actor nodes with minimum spectrum access. Effects of licensed user in terms of both interruption and interference are modeled, and implications on design and performance are highlighted. Proposed scheme is shown to be satisfying consensus convergence requirements, especially in dense licensed user activity and high interference scenarios which can be encountered in various portions of the Smart Grid. Some of the future research directions can be listed as investigation of the effects PU mobility on opportunistic consensus and comparison of consensus results under different PU channel occupancy models.

\section{References}

[1] I. F. Akyildiz, W. Su, Y. Sankarasubramaniam, and E. Cayirci, "A survey on Sensor Networks," IEEE Communications Magazine, vol. 40, no. 8, pp. 102-114, Aug. 2002.

[2] I. F. Akyildiz, and I. H. Kasimoglu, "Wireless sensor and actor networks: research challenges," Ad Hoc Networks (Elsevier), vol .2, no. 4, pp. 351367, Oct. 2004.

[3] I. F. Akyildiz, W. Y. Lee, M. C. Vuran, and S. Mohanty, "NeXt Generation/Dynamic Spectrum Access/Cognitive Radio Wireless Networks: A Survey," Comput. Netw. J., vol. 50, no. 13, pp. 2127-2159, 2006.

[4] O. B. Akan, O. B. Karli, and O. Ergul, "Cognitive Radio Sensor Networks," IEEE Network, vol. 23, no.4, pp. 34-40, July-August 2009.

[5] R. Oflati-Saber, J. A. Fax, and R. M. Murray, "Consensus and Cooperation in Networked Multi-Agent Systems," Proceedings of the IEEE, vol. 95, no. 1, pp. 215-233 Jan. 2007.

[6] J. A. Han, W. S. Jeon, and D. G. Jeong, "Energy-Efficient Channel Management Scheme for Cognitive Radio Sensor Networks," IEEE Trans. on Vehicular Tech., vol. 60, no. 4, pp. 1905-1910, May 2011.

[7] X. Li, D. Wang, J. McNair, and J. Chen, "Residual Energy Aware Channel Assignment in Cognitive Radio Sensor Networks," in in Proc. IEEE WCNC 2011., 2011.

[8] S. Maleki, A. Pandharipande, and G. Leus, "Energy-Efficient Distributed Spectrum Sensing for Cognitive Sensor Networks," IEEE Sensors Journal, vol. 11, no. 3, pp. 565-573, 2011.

[9] M. C. Vuran and I. F. Akyildiz, "Error Control in Wireless Sensor Networks: A Cross Layer Analysis," IEEE/ACM Transactions on Networking, vol. 17, no. 4, pp. 1186-1199, Aug. 2009.

[10] M. C. Vuran and I. F. Akyildiz, "Spatial Correlation-based Collaborative Medium Access Control in Wireless Sensor Networks," IEEE/ACM Transactions on Networking, vol. 14, no. 2, pp. 316 -329, April 2006.

[11] M. C. Vuran O. B. Akan, I. F. Akyildiz, "Spatio-Temporal Correlation: Theory and Applications for Wireless Sensor Networks," Computer Networks Journal (Elsevier), vol. 45, no 3, pp. 245 -259, June 2004.

[12] Y. T. Hou, Y. Shi, and H. D. Sherali, "Spectrum Sharing for Multi-Hop Networking with Cognitive Radios," IEEE Journal of Selected Areas in Communications, vol. 26, no. 1, pp. 146-155, Jan. 2008.
[13] R. Zhang, and Y.-C. Liang, "Exploiting Multi-Antennas for Opportunistic Spectrum Sharing in Cognitive Radio Networks," IEEE Journal of Selected Topics in Signal Processing, vol. 2, no. 1, pp. 88-

[14] L. Zhang, Y. Xin, and Y.-C. Liang, "Weighted Sum Rate Optimization for Cognitive Radio MIMO Broadcast Channels," IEEE Trans. on Wireless Commun., vol. 8, no. 6, pp. , July 2009.

[15] K. Hamdi, W. Zhang, and K. B. Letaief, "Opportunistic Spectrum Sharing in Cognitive MIMO Wireless Networks," IEEE Trans. on Wireless Commun., vol 8, no. 8, August 2009.

[16] C. Gao, Y. Shi, Y. T. Hou, and S. Kompella, "On the Throughput of MIMO-Empowered Multihop Cognitive Radio Networks," IEEE Trans. on Mobile Computing, vol. 10, no. 11, pp. , Nov. 2011.

[17] G. A. Shah, V. C. Gungor, O. B. Akan, "A Cross-Layer QoS-Aware Communication Framework in Cognitive Radio Sensor Networks for Smart Grid Applications," IEEE Transactions on Industrial Informatics, vol.9, no.3, pp.1477,1485, Aug. 2013.

[18] J. Huang, H. Wang, Y. Qian, C. Wang, "Priority-Based Traffic Scheduling and Utility Optimization for Cognitive Radio Communication Infrastructure-Based Smart Grid," IEEE Transactions on Smart Grid, vol.4, no.1, pp.78,86, March 2013.

[19] R. Yu, Y. Zhang, S. Gjessing, Y. Chau, S. Xie, M. Guizani, "Cognitive radio based hierarchical communications infrastructure for smart grid," IEEE Network, vol.25, no.5, pp.6,14, September-October 2011.

[20] R. Deng, J. Chen, X. Cao, Y. Zhang, S. Maharjan, S. Gjessing, "SensingPerformance Tradeoff in Cognitive Radio Enabled Smart Grid," IEEE Transactions on Smart Grid, vol.4, no.1, pp.302,310, March 2013.

[21] H. Wang, Y. Qian, H. Sharif, "Multimedia communications over cognitive radio networks for smart grid applications," IEEE Wireless Communications, vol.20, no.4, pp.125,132, August 2013.

[22] S. Bu, F. R. Yu, "Green Cognitive Mobile Networks With Small Cells for Multimedia Communications in the Smart Grid Environment," IEEE Transactions on Vehicular Technology, vol.63, no.5, pp.2115,2126, Jun 2014.

[23] K. Sriram and W. Whitt, "Characterizing superposition arrival processes in packet multiplexer for voice and data," IEEE J. Sel. Areas Commun., vol. 4, no. 6, pp. 833846, Sep. 1986.

[24] R. Urgaonkar and M. J. Neely, "Opportunistic scheduling with reliability guarantees in cognitive radio network," in Proc. IEEE INFOCOM 2008, 2008 .

[25] S. Huang, X. Liu, and Z. Ding, "Opportunistic spectrum access in cognitive radio networks," in Proc. IEEE INFOCOM 2008, pp. 1734-1743, 2008.

[26] W. Y. Lee, and I. F. Akyildiz, "Optimal Spectrum Sensing Framework for Cognitive Radio Networks,” IEEE Trans. on Wireless Commun., vol. 7, no. 10 , pp. 3845-3857, 2008.

[27] M. Zuniga, B. Krishnamachari, "Analyzing the Transitional Region in Low Power Wireless Links," in Proc. IEEE SECON, pp. 517-526, 2004.

[28] S. Lin, and D. J. Costello Jr., Error Control Coding: Fundamentals and Applications. Englewood Cliffs, NJ: Prentice-Hall, 1983.

[29] J. M. Mendel, Lessons in Estimation Theory for Signal Processing, Communications, and Control.Englewood Cliffs, NJ: Prentice-Hall, 1995.

[30] P. C. Gilmore, and R. E. Gomory, "A linear programming approach to the cutting stock problem", Operations Research, vol. 9, no. 6, pp. 848-859, Nov. - Dec. 1961.

[31] P. C. Gilmore, and R. E. Gomory, "A linear programming approach to the cutting stock problem-Part II," Operations Research, vol. 11, no. 6, pp. 863-888, Nov. - Dec. 1963.

[32] IBM ILOG CPLEX V12.1: Users Manual for CPLEX, International Business Machines Corporation, 2009.

[33] S. Sardellitti, S. Barbarossa, and A. Swami, "Optimal Topology Control and Power Allocation for Minimum Energy Consumption in Consensus Networks," IEEE Trans. on Signal Processing, vol. 60, no. 1, pp. 383-399, Jan. 2012

[34] C. G. Lopes, and A. H. Sayed, "Diffusion Least-Mean Squares Over Adaptive Networks: Formulation and Performance Analysis," IEEE Trans. on Signal Processing, vol. 56, no. 7, pp. 3122-3136, July 2008. 